\title{
Isolated high tibial osteotomy is appropriate in less than two-thirds of varus knees if excessive overcorrection of the medial proximal tibial angle should be avoided
}

\author{
Matthias J. Feucht ${ }^{1,2}$ (D) Philipp W. Winkler ${ }^{1}$. Julian Mehl ${ }^{1} \cdot$ Gerrit Bode $^{2} \cdot$ Philipp Forkel $^{1}$ - Andreas B. Imhoff ${ }^{1}$. \\ Patricia M. Lutz ${ }^{1}$
}

Received: 30 May 2020 / Accepted: 14 July 2020 / Published online: 20 July 2020

(c) The Author(s) 2020

\begin{abstract}
Purpose To perform a detailed deformity analysis of patients with varus alignment and to define the ideal osteotomy level (tibial vs. femoral vs. double level) to avoid an oblique joint line.

Methods A total of 303 digital full-leg standing radiographs of patients aged 18-60 years and varus alignment [mechanical tibiofemoral varus angle $(\mathrm{mFTA}) \geq 3^{\circ}$ ] were included. All legs were analyzed regarding $\mathrm{mFTA}$, mechanical medial proximal tibia angle (mMPTA), mechanical lateral distal femur angle (mLDFA), and joint line convergence angle. Based on mFTA, varus alignment was categorized as "mild" $\left(3^{\circ}-5^{\circ}\right)$, "moderate" $\left(6^{\circ}-8^{\circ}\right)$, or "severe" $\left(\geq 9^{\circ}\right)$. Deformity location was determined according to the malalignment test described by Paley. Two osteotomy simulations were performed with different upper limits for mMPTA: anatomic correction (mMPTA $\leq 90^{\circ}, \mathrm{mLDFA} \geq 85^{\circ}$ ) and overcorrection (mMPTA $\leq 95^{\circ}$, mLDFA $\geq 85^{\circ}$ ). If a single osteotomy exceeded these limits at the intended mFTA of $2^{\circ}$ valgus, a double-level osteotomy was simulated. If even a double-level osteotomy resulted in deviations from the defined limits, the leg was categorized as "uncorrectable".

Results Mean mFTA was $6^{\circ} \pm 11^{\circ}$ of varus (range $3^{\circ}-15^{\circ}$ ). A tibial deformity was observed in $28 \%$, a femoral deformity in $23 \%$, a combined tibial and femoral deformity in $4 \%$, and no bony deformity in $45 \%$. The prevalence of a tibial deformity did not differ between varus severity groups, whereas a femoral and bifocal deformity was significantly more prevalent in knees with more distinct varus $(p<0.001)$. Osteotomy simulation revealed that isolated high tibial osteotomy (HTO) was appropriate in only $12 \%$ for anatomic correction, whereas a double-level osteotomy was necessary in $63 \%$. If overcorrection of mMPTA was tolerated, the number of HTOs significantly increased to 57\% $(p<0.001)$, whereas the number of doublelevel osteotomies significantly decreased to $33 \%(p<0.001)$. Isolated DFO was considered ideal in $8 \%$ for both simulations. Significantly more knees were considered "uncorrectable" by simulating anatomic correction $(18$ vs. $2 \% ; p<0.001)$. A double-level osteotomy was significantly more often necessary in knees with "severe" varus $(p<0.001)$.

Conclusion Less than one-third of patients (28\%) with mechanical varus $\geq 3^{\circ}$ have a tibial deformity. If anatomic correction $\left(\mathrm{mMPTA} \leq 90^{\circ}\right)$ is intended, only $12 \%$ of patients can be corrected via isolated HTO, whereas $63 \%$ of patients require a double-level osteotomy. If slight overcorrection is accepted (mMPTA $\left.\leq 95^{\circ}\right), 57 \%$ of patients can be corrected via isolated HTO, whereas $33 \%$ of patients would still require a double-level osteotomy.
\end{abstract}

Level of evidence III, cross-sectional study.

Keywords Varus $\cdot$ Osteotomy $\cdot$ HTO $\cdot$ Malalignment $\cdot$ Alignment

The research was performed at the Department for Orthopedic Sports Medicine, Technical University Munich, Germany.

Matthias J. Feucht

matthias.feucht@gmx.net

Extended author information available on the last page of the article

\section{Introduction}

Varus malalignment has historically been considered a tibial-based deformity and the broad majority of varus deformities are corrected via high tibial osteotomy (HTO) [3, 10, 19, 26]. However, recent studies have found highly variable coronal alignment in both, osteoarthritic and non-osteoarthritic 
knees [21, 23, 25, 34]. Based on these studies, varus malalignment can be the result of a tibial deformity, a femoral deformity, or a combined femoral and tibial deformity. Furthermore, varus malalignment may occur due to intraarticular wear and/or lateral ligament laxity without the presence of a bony deformity. Following the basic principles described by Dror Paley [39], osteotomies should be performed at the location of the deformity. If this rule is ignored, corrective osteotomies can result in an oblique joint line, which has been shown to negatively affect functional outcomes and survival after HTO [1, 4, 11, 47]. More specifically, if valgus HTO is performed in a normally aligned tibia, overcorrection of the mechanical medial proximal tibial angle (mMPTA) results in pathologic lateral inclination of the joint line [5, 39]. Excessive overcorrection of the mMPTA should be avoided, since studies have shown that a postoperative mMPTA of greater $95^{\circ}$ leads to increased shear stress in the medial compartment and inferior clinical outcome $[1,37,47]$. Therefore, a femoral osteotomy [16, 51] or a combined tibial and femoral osteotomy (double-level osteotomy [5, 36, 41, 42, 45]) may be necessary in several patients to avoid an oblique joint line.

In a previous study, Eberbach et al. [13] have analyzed the geometry of valgus knees and found that a tibial deformity was most common, followed by a combined femoral and tibial deformity. Similar studies for varus knees are lacking. The purpose of this study was to perform a detailed deformity analysis of patients with varus malalignment and to define the ideal osteotomy level to avoid an oblique joint line. The hypothesis was that a femoral or bifocal deformity is observed in a relevant number of patients with varus malalignment and that a femoral or double-level osteotomy would be necessary in several patients to avoid an oblique joint line.

\section{Materials and methods}

All digital full-leg standing radiographs performed at the authors institution between 2017 and 2019 were reviewed for potential inclusion. For the purpose of this study, only subjects with significant varus alignment, defined as a mechanical tibiofemoral varus angle (mFTA) of $\geq 3^{\circ}[39$, 48], were included. Further inclusion criteria were: Male and female patients, age 18-60 years, and osteoarthritis Grad $0-I V$ according to Kellgren and Lawrence [27]. Exclusion criteria were: Skeletal immaturity with open growth plates, posttraumatic deformities, previous surgery affecting limb alignment, previous hip, knee, or ankle replacement, and malrotated radiographs with a decentralized patella.

Computer-based deformity analysis and osteotomy simulation was performed using a commercially available planning software (mediCAD ${ }^{\circledR}$, Hectec GmbH, Germany). This software allows for precise analysis of the alignment as well as simulation of single and multiple osteotomies with a high intra- and interrater reliability $[18,44]$. All measurements were done by a single observer. Intra- and interrater reliability testing was conducted on 20 randomly chosen and blinded subjects after an interval of 3 weeks.

\section{Deformity analysis}

All digital radiographs were imported to the mediCAD ${ }^{\circledR}$ program and calibrated. Necessary landmarks were marked, including the center of the femoral head, the apex of the greater trochanter, femoral and tibial knee base, medial and lateral border of the femoral condyles and tibial plateau, medial and lateral border of the talus, and the joint line of the talus. Based on these landmarks, all relevant parameters are calculated automatically by the software. For the purpose of this study, the following parameters were recorded: mechanical femorotibial angle (mFTA), position of the weight bearing line (WBL ratio; expressed as \% of the medial-to-lateral width of the tibial plateau), mechanical medial proximal tibia angle (mMPTA), mechanical lateral distal femur angle (mLDFA), and joint line convergence angle (JLCA). Based on the amount of varus malalignment, patients were categorized as "mild" $\left(3^{\circ}-5^{\circ}\right.$ varus), "moderate" ( $6^{\circ}-8^{\circ}$ varus), or "severe" ( $\geq 9^{\circ}$ varus).

Next, the malalignment test as described by Paley et al. [39] was performed to determine the location of the deformity, with normal values for mMPTA and mLDFA of $85^{\circ}-90^{\circ}$ [40]. Based on the deformity location, patients were assorted to one of the following 4 groups:

- Tibial deformity: mMPTA $<85^{\circ}$, mLDFA normal

- Femoral deformity: mLDFA $>90^{\circ}$, mMPTA normal

- Tibial + femoral deformity: mMPTA $<85^{\circ}$ and mLDFA $>90^{\circ}$

- No bony deformity: mMPTA and mLDFA normal

Given a considerable number of patients without a true bony deformity as defined above, patients were further analyzed regarding their potential for bony correction based on deviations from the upper or lower limit of the MMPTA and mLDFA, respectively:

- Tibial potential: $\mathrm{mMPTA}<90^{\circ}$

- Femoral potential: mLDFA $>85^{\circ}$

- Tibial and femoral potential: mMPTA $<90^{\circ}$ and mLDFA $>85^{\circ}$

- No potential: $\mathrm{mMPTA} \geq 90^{\circ}$ and $\mathrm{mLDFA} \leq 85^{\circ}$ 


\section{Osteotomy simulation}

Simulation of the osteotomy always started at the site of the greatest deformity as revealed in the malalignment test: In case of a tibial-based deformity, a medial openwedge HTO was simulated and in case of a femoral-based deformity a lateral closed-wedge DFO was simulated. If no true bony deformity was present, the primary osteotomy site was chosen based on the greatest amount of bony correction potential. All legs were corrected to a postoperative mFTA of $2^{\circ}$ valgus [50]. To avoid an oblique joint line, limits for the postoperative knee base angles were defined. For the mLDFA, the postoperative lower limit was set at $85^{\circ}$ for all simulations [36]. With regard to the mMPTA, two different simulations were performed: one simulation with a postoperative upper limit of $90^{\circ}$ (anatomic correction) and another simulation with a postoperative upper limit of $95^{\circ}$ (overcorrection). If the simulation of a single osteotomy (HTO or DFO) led to deviation from these limits at the intended mFTA of $2^{\circ}$ valgus, a doublelevel osteotomy was simulated. In this case, the first osteotomy was simulated at the site of the greatest deformity and the corresponding knee base angle was corrected to its upper or lower limit, respectively. The second osteotomy was simulated at the opposite site until the intended alignment of $2^{\circ}$ of mechanical valgus was achieved. Based on these simulations, the ideal osteotomy level was classified as "tibial", "femoral", or "double-level". If even a double-level osteotomy resulted in deviations from the defined limits for knee base angles, the leg was categorized as "uncorrectable". Illustrative case examples are shown in Figs. 1, 2, 3.

\section{Statistical analysis}

Statistical analysis was performed using SPSS software version 25.0 (IBM-SPSS, New York, USA). Continuous variables were calculated as mean \pm standard deviation and categorical variables as count and percentages.

Intraclass correlation coefficients (ICCs) were calculated to determine the intra- and interobserver reproducibility of the obtained measurements.

Normal distribution of all data was evaluated with the Kolmogorov-Smirnov test.

Distribution of deformity location and ideal osteotomy level with regard to the amount of varus malalignment ("mild" vs. "moderate" vs. "severe") and osteotomy simulation (anatomic vs. overcorrection) was compared with the Qui-square test followed by post hoc tests with Bonferroni correction of the $p$ value. JLCA between knees with "mild", "moderate", or "severe" varus was compared with the Kruskal-Wallis test followed by post hoc analysis with Bonferroni correction.

This study was approved by the Ethics Committee of the Technical University of Munich.

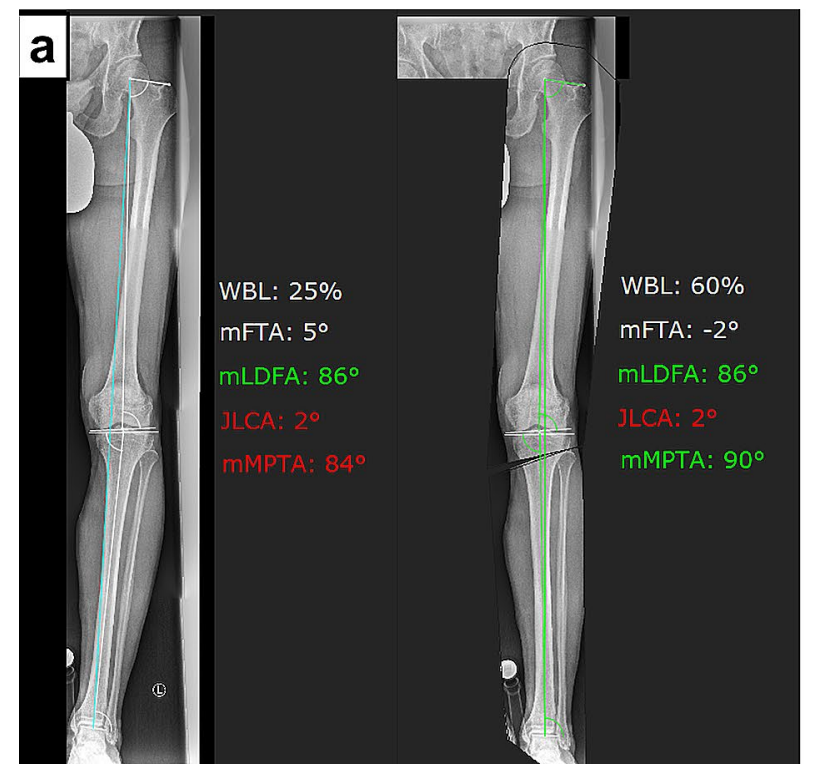

Fig. 1 Illustrative case examples 1 and 2. a Case 1: deformity analysis revealed a tibial-based varus deformity of $5^{\circ}$ with a normal mLDFA and a pathologic mMPTA. This deformity can be corrected via medial open-wedge HTO to the desired alignment of $2^{\circ}$ of valgus without exceeding the upper limit of the mMPTA. b Case 2: deform-

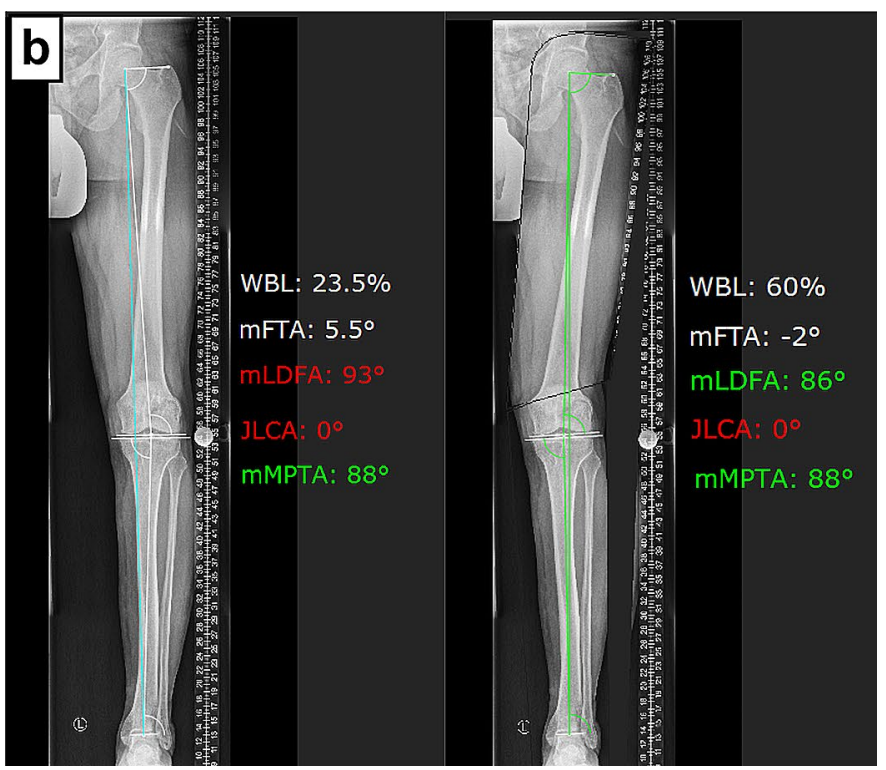

ity analysis revealed a femoral-based varus deformity of $5.5^{\circ}$ with a normal mMPTA and a pathologic mLDFA. This deformity can be corrected via lateral closed-wedge DFO to the desired alignment of $2^{\circ}$ of valgus without exceeding the lower limit of the mLDFA 

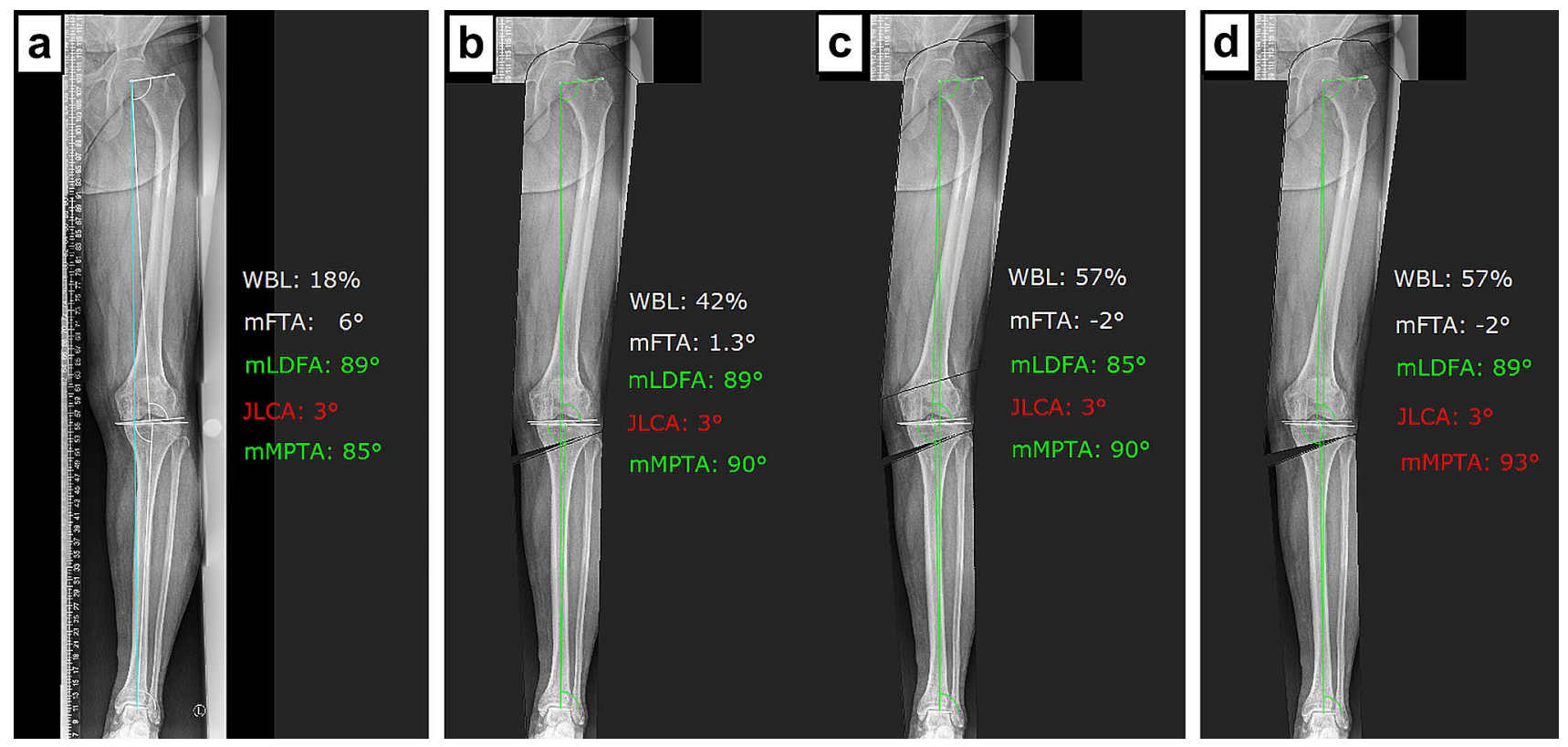

Fig. 2 Illustrative case example 3. a Deformity analysis revealed a varus deformity of $6^{\circ}$ without a true bone deformity based on the malalignment test [39]. However, potential for bony correction exist in both, the proximal tibia and distal femur, with the greater potential being located at the proximal tibia. b, c First osteotomy simulation tolerating mLDFA $\geq 85^{\circ}$ and $\mathrm{mMPTA} \leq 90^{\circ}$ (anatomic correction): by simulating HTO alone, $1.3^{\circ}$ of varus alignment remains with the mMPTA set at $90^{\circ}$. By simulating a double-level osteotomy, the deformity can be corrected to the desired alignment of $2^{\circ}$ of valgus without exceeding the upper and lower limit of the mMPTA and mLDFA, respectively. d Second osteotomy simulation tolerating mLDFA $\geq 85^{\circ}$ and $\mathrm{mMPTA} \leq 95^{\circ}$ (overcorrection): the deformity can be corrected via HTO to the desired alignment of $2^{\circ}$ of valgus without exceeding the upper limit of the mMPTA of $95^{\circ}$
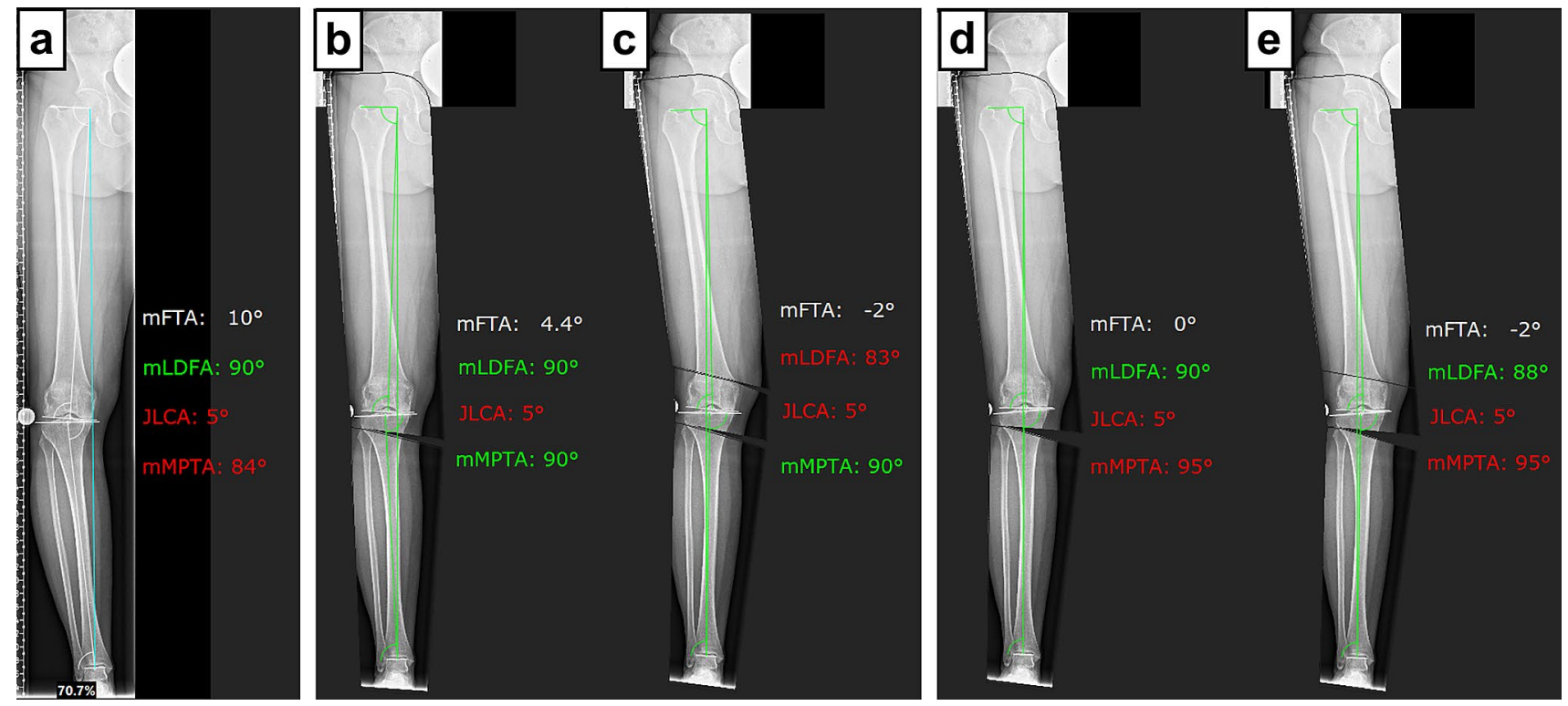

Fig. 3 Illustrative case example 4. a Deformity analysis revealed a tibial-based varus deformity of $10^{\circ}$ with a high-normal mLDFA and a pathologic mMPTA. b, c First osteotomy simulation tolerating mLDFA $\geq 85^{\circ}$ and $\mathrm{mMPTA} \leq 90^{\circ}$ (anatomic correction): by simulating HTO alone, $4.4^{\circ}$ of varus alignment remains with the mMPTA set at $90^{\circ}$. By simulating a double-level osteotomy to the desired alignment of $2^{\circ}$ of valgus, the lower limit of the mLDFA is exceeded. This case is, therefore, considered "uncorrectable". d, e Second osteotomy simulation tolerating mLDFA $\geq 85^{\circ}$ and $\mathrm{mMPTA} \leq 95^{\circ}$ (overcorrection): by simulating HTO alone, neutral alignment remains with the mMPTA set at $95^{\circ}$. By simulating a double-level osteotomy, the deformity can be corrected to the desired alignment of $2^{\circ}$ of valgus without exceeding the upper and lower limit of the mMPTA and mLDFA, respectively 


\section{Results}

A total of 303 full-leg standing radiographs could be included. Patient demographics are provided in Table 1.

\section{Deformity analysis}

Measurements of the deformity analysis and corresponding ICC values are shown in Table 2. For the total study population, the malalignment test revealed a tibial deformity in $28 \%$, a femoral deformity in $23 \%$, a combined tibial and femoral deformity in $4 \%$, and no bony deformity in $45 \%$ (Fig. 4). Potential for bony correction was observed in all patients, with almost all patients demonstrating potential for correction at the tibial and femoral site $(94 \%)$. The greatest

Table 1 Patient demographics of the total study group

\begin{tabular}{ll}
\hline Number of patients & 303 \\
\hline Sex & \\
Female & $24 \%(72)$ \\
Male & $76 \%(231)$ \\
Age (years) & $44 \pm 11(18-60)$ \\
Laterality & \\
Left & $55 \%(165)$ \\
Right & $46 \%(138)$ \\
Osteoarthritis according to Kellgren and Lawrence & \\
No OA & $11 \%(34)$ \\
Grade I & $34 \%(104)$ \\
Grade II & $29 \%(88)$ \\
Grade III & $18 \%(53)$ \\
Grade IV & $8 \%(24)$ \\
Varus deformity & \\
Mild $\left(3^{\circ}-5^{\circ}\right)$ & $59 \%(178)$ \\
Moderate $\left(6^{\circ}-8^{\circ}\right)$ & $32 \%(98)$ \\
Severe $\left(\geq 9^{\circ}\right)$ & $9 \%(27)$ \\
\hline
\end{tabular}

Continuous variables are shown as mean \pm standard deviation and (range), categorical variables are shown as percentages per group and (number of patients) potential for correction was observed at the tibial site in $56 \%$.

Deformity location with regard to the amount of varus malalignment is summarized in Table 3. No significant difference was observed between the three groups ("mild" vs. "moderate" vs. "severe") for the prevalence of a tibial deformity. Compared to "mild" varus, a femoral deformity was significantly more prevalent in "moderate" and "severe" varus ( $p=0.016$ and $p=0.008)$, and a combined femoral + tibial deformity was significantly more prevalent in "severe" varus as compared to "mild" and "moderate" varus $(p<0.001$ and $p=0.011)$. No bony deformity was significantly more prevalent in "mild" varus as compared to "moderate" and "severe" varus ( $p<0.001)$, and significantly more prevalent in "moderate" varus as compared to "severe" varus $(p=0.034)$. Comparison of JLCA between the three groups revealed a significantly higher JLCA in "moderate" and "severe" varus as compared to "mild" varus $(p=0.002$ and $p<0.001)$.

\section{Osteotomy simulation}

Distribution of the ideal osteotomy level for both simulations (mMPTA $\leq 90^{\circ}$ and $\mathrm{mMPTA} \leq 95^{\circ}$ ) is shown in Fig. 5. An isolated HTO was appropriate in $12 \%$ for an anatomic correction, whereas a double-level osteotomy was necessary in $63 \%$. If overcorrection was tolerated, the number of HTOs significantly increased to $57 \%(p<0.001)$, whereas the number of double-level osteotomies significantly decreased to $33 \%(p<0.001)$. An isolated DFO was considered ideal in $8 \%$ for both simulations.

Distribution of the ideal osteotomy level with regard to the amount of varus malalignment is summarized in Tables 4 and 5. By simulating an anatomic correction, HTO was significantly more often appropriate in knees with "mild" varus, as compared to knees with "moderate" or "severe" varus $(p<0.001)$. Knees with "severe" varus were significantly more frequently considered uncorrectable, as compared to knees with "mild" or "moderate" varus $(p<0.001$ and $p=0.034)$. By simulating overcorrection of the mMPTA, HTO was significantly more often
Table 2 Measurements of the deformity analysis and corresponding intraclass correlation coefficients

\begin{tabular}{lcllll}
\hline & Mean \pm SD & Median & Range & Intrarater ICC & Interrater ICC \\
\hline mFTA & $6^{\circ} \pm 11^{\circ}$ & $5^{\circ}$ & $3^{\circ}-15^{\circ}$ & 0.997 & 0.996 \\
WBL ratio & $23 \pm 8 \%$ & $24 \%$ & $1-39 \%$ & 0.994 & 0.992 \\
JLCA & $2^{\circ} \pm 2^{\circ}$ & $2^{\circ}$ & $0^{\circ}-8^{\circ}$ & 0.903 & 0.940 \\
mMPTA & $86^{\circ} \pm 2^{\circ}$ & $86^{\circ}$ & $78^{\circ}-93^{\circ}$ & 0.991 & 0.984 \\
mLDFA & $89^{\circ} \pm 2^{\circ}$ & $89^{\circ}$ & $83^{\circ}-95^{\circ}$ & 0.981 & 0.965 \\
\hline
\end{tabular}

$S D$ standard deviation, $I C C$ intraclass correlation coefficient, $m F T A$ mechanical femorotibial angle, $W B L$ weight bearing line, JLCA joint line convergence angle, $M M P T A$ mechanical medial proximal tibial angle, $m L D F A$ mechanical lateral distal femoral angle 


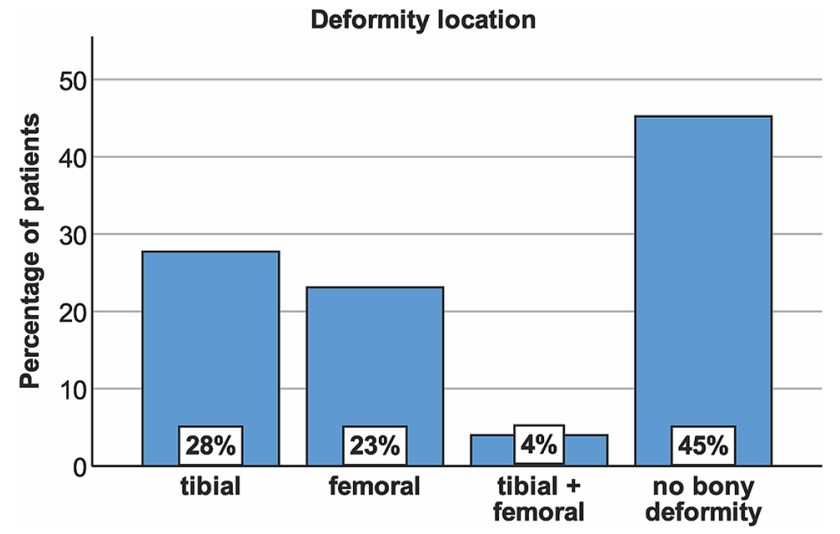

Fig. 4 Deformity location based on the malalignment test [39] with normal values for mMPTA and mLDFA of $85^{\circ}-90^{\circ}$ [40]. Tibial deformity: $\mathrm{mMPTA}<85^{\circ}$, mLDFA normal; femoral deformity: mLDFA $>90^{\circ}$, mMPTA normal; tibial + femoral deformity: mMPTA $<85^{\circ}+$ mLDFA $>90^{\circ}$; no bony deformity: mMPTA + mLDFA normal

appropriate in knees with "mild" and "moderate" varus, as compared to knees with "severe" varus $(p<0.001$ and $p=0.005$ ). Compared to "mild" and "moderate" varus, a double-level osteotomy was significantly more often necessary in knees with "severe" varus $(p<0.001$ and $p=0.001$ ).

\section{Discussion}

The most important findings of the present study were that only $28 \%$ of patients with varus malalignment $\geq 3^{\circ}$ had a tibial deformity based on the malalignment test described by Paley. Most patients (45\%) had no bony deformity, and another $23 \%$ had a femoral deformity. If anatomic correction $\left(\mathrm{mMPTA} \leq 90^{\circ}, \mathrm{mLDFA} \geq 85^{\circ}\right.$ ) was intended, only $12 \%$ of patients could be corrected via isolated HTO, whereas $63 \%$ of patients required a double-level osteotomy. If slight overcorrection at the tibial site was accepted (mMPTA $\leq 95^{\circ}$ ), $57 \%$ of patients could be corrected via isolated HTO, whereas $33 \%$ of patients still required a double-level osteotomy, and $8 \%$ were best corrected via isolated DFO.

Valgus-producing HTO has been used for several decades as a surgical treatment for medial compartment OA associated with varus malalignment $[10,11]$. With continuous improvements in surgical technique and the introduction of angle-stable implants, indications for corrective osteotomies have been extend [15, 30], and HTO is nowadays regularly performed as a concomitant procedure in patients undergoing cartilage repair procedures $[6,32,33]$, meniscal transplantation [52], or ligament reconstruction [49]. Most clinical outcome data are available for the treatment of medial compartment OA, and valgus HTO can be considered an evidence-based procedure [9]. Several negative predictive factors for worse outcome and failure have been reported including increased age, high BMI, advanced OA,

Table 3 Deformity location based on the malalignment test [39] with regard to the amount of varus malalignment

\begin{tabular}{llll}
\hline & \multicolumn{2}{l}{ Varus malalignment $(\mathrm{mFTA})$} & \\
\cline { 2 - 3 } & Mild $\left(3^{\circ}-5^{\circ}\right)$ & Moderate $\left(6^{\circ}-8^{\circ}\right)$ & Severe $\left(\geq 9^{\circ}\right)$ \\
\hline Deformity location & & & $33 \%$ \\
Tibial (mMPTA $<85^{\circ}$, mLDFA normal) & $23 \%$ & $35 \%$ & $41 \%^{\mathrm{a}}$ \\
Femoral (mLDFA $>90^{\circ}$, mMPTA normal) & $16 \%$ & $31 \%^{\mathrm{a}}$ & $19 \%^{\mathrm{b}}$ \\
Tibial + femoral (mMPTA $<85^{\circ}+$ mLDFA $\left.>90^{\circ}\right)$ & $2 \%$ & $3 \%$ & $7 \%^{\mathrm{d}}$ \\
No deformity (mMPTA + mLDFA normal) & $58 \%^{\mathrm{c}}$ & $32 \%^{\mathrm{d}}$ & $3.3^{\circ} \pm 1.8^{\mathrm{e}}\left(0.2^{\circ}-6.8^{\circ}\right)$ \\
JLCA & $1.8^{\circ} \pm 1.3^{\circ}\left(0.1^{\circ}-6.4^{\circ}\right)$ & $2.4^{\circ} \pm 1.6^{\mathrm{e}}\left(0.1^{\circ}-7.7^{\circ}\right)$ & \\
\hline
\end{tabular}

Normal values for mMPTA and mLDFA were $85^{\circ}-90^{\circ}[39,40]$

Values are shown as percentages per group or mean \pm standard deviation and range

mFTA mechanical femorotibial angle, $m M P T A$ mechanical medial proximal tibial angle, $m L D F A$ mechanical lateral distal femoral angle, $J L C A$ joint line convergence angle

${ }^{a}$ Significant difference between $3^{\circ}-5^{\circ}$ and $6^{\circ}-8^{\circ} \mathrm{mFTA}(p=0.016)$ and between $3^{\circ}-5^{\circ}$ and $\geq 9^{\circ} \mathrm{mFTA}(p=0.008)$ (Qui-square test followed by post hoc tests with Bonferroni correction)

${ }^{\mathrm{b}}$ Significant difference compared to $3^{\circ}-5^{\circ}$ and $6^{\circ}-8^{\circ} \mathrm{mFTA}(p<0.001$ and $p=0.011)$ (Qui-square test followed by post hoc tests with Bonferroni correction)

${ }^{\mathrm{c}}$ Significant difference compared to $6^{\circ}-8^{\circ}$ and $\geq 9^{\circ} \mathrm{mFTA}(p<0.001)$ (Qui-square test followed by post hoc tests with Bonferroni correction)

${ }^{\mathrm{d}}$ Significant difference compared to $\geq 9^{\circ} \mathrm{mFTA}(p=0.034)$ (Qui-square test followed by post hoc tests with Bonferroni correction)

${ }^{\text {e }}$ Significant difference between $3^{\circ}-5^{\circ}$ and $6^{\circ}-8^{\circ} \mathrm{mFTA}(p=0.002)$ and between $3^{\circ}-5^{\circ}$ and $\geq 9^{\circ} \mathrm{mFTA}(p<0.001)$ (Kruskal-Wallis test followed by post hoc analysis with Bonferroni correction) 
Fig. 5 Ideal osteotomy level tolerating a mechanical medial proximal tibial angle (mMPTA) of $\leq 90^{\circ}$ (anatomic correction) or $\leq 95^{\circ}$ (overcorrection). \#1 significant difference compared to $\mathrm{mMPTA} \leq 90^{\circ}(p<0.001)$; $\# 2$ significant difference compared to $\mathrm{mMPTA} \leq 95^{\circ}$ $(p<0.001)$; \#3 significant difference compared to mMPTA $\leq 95^{\circ}(p<0.001)$ (Qui-square test followed by post hoc tests with Bonferroni correction)

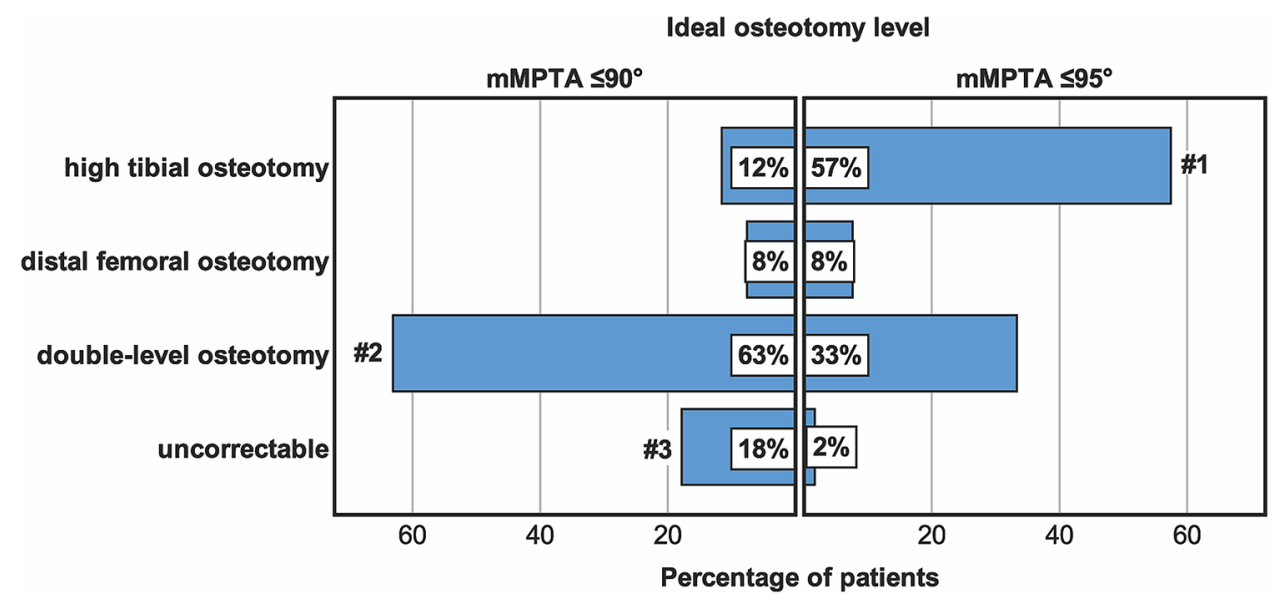

Table 4 Ideal osteotomy level tolerating a mechanical lateral distal femoral angle (mLDFA) of $\geq 85^{\circ}$ and a mechanical medial proximal tibial angle (mMPTA) of $\leq 90^{\circ}$ (anatomic correction) with regard to the amount of varus malalignment

\begin{tabular}{llll}
\hline Ideal osteotomy level & \multicolumn{3}{l}{ Varus malalignment (mFTA) } \\
\cline { 2 - 4 } & $\begin{array}{l}\text { Mild }\left(3^{\circ}-5^{\circ}\right) \\
(\%)\end{array}$ & $\begin{array}{l}\text { Moderate } \\
\left(6^{\circ}-8^{\circ}\right)(\%)\end{array}$ & $\begin{array}{l}\text { Severe } \\
\left(\geq 9^{\circ}\right) \\
(\%)\end{array}$ \\
\hline Tibial & $18^{\mathrm{a}}$ & 3 & 0 \\
Femoral & 11 & 4 & 0 \\
Double-level & 59 & 72 & 56 \\
Uncorrectable & 12 & 20 & $44^{\mathrm{b}}$ \\
\hline
\end{tabular}

Values are shown as percentages per group

mFTA mechanical femorotibial angle

${ }^{\text {a }}$ Significant difference compared to $6^{\circ}-8^{\circ}$ and $\geq 9^{\circ}$ mFTA $(p<0.001)$ (Qui-square test followed by post hoc tests with Bonferroni correction)

${ }^{\mathrm{b}}$ Significant difference compared to $3^{\circ}-5^{\circ}$ and $6^{\circ}-8^{\circ}(p<0.001$ and $p=0.034$ ) (Qui-square test followed by post hoc tests with Bonferroni correction)

and under- or overcorrection [8, 14, 24]. Another important factor to consider is postoperative joint line obliquity $[4,5$, 11]. This phenomenon can occur if HTO is performed in a normally aligned tibia, or if a severe varus deformity is corrected via HTO alone [36, 37]. Beside difficulties in converting to total knee arthroplasty [20], an oblique joint line and particularly excessive overcorrection of the mMPTA has been associated with worse clinical outcome and higher failure rates after HTO [1, 4, 11, 47]. However, no consensus exists to what extent overcorrection of the MMPTA is acceptable [17]. Using a 3D finite element model analysis, Nakayama et al. [37] could demonstrate that HTO induced excessive shear stress in the medial compartment if joint line obliquity was $5^{\circ}$ or more. The authors, therefore, proposed a double-level osteotomy in varus knees with a preoperatively anticipated mMPTA $>95^{\circ}$ [37]. This proposal is affirmed
Table 5 Ideal osteotomy level tolerating a mechanical lateral distal femoral angle (mLDFA) of $\geq 85^{\circ}$ and a mechanical medial proximal tibial angle (mMPTA) of $\leq 95^{\circ}$ (overcorrection) with regard to the amount of varus malalignment

\begin{tabular}{lllc}
\hline Ideal osteotomy level & \multicolumn{3}{l}{ Varus malalignment (mFTA) } \\
\cline { 2 - 4 } & $\begin{array}{l}\text { Mild }\left(3^{\circ}-5^{\circ}\right) \\
(\%)\end{array}$ & $\begin{array}{l}\text { Moderate } \\
\left(6^{\circ}-8^{\circ}\right)(\%)\end{array}$ & $\begin{array}{l}\text { Severe } \\
\left(\geq 9^{\circ}\right) \\
(\%)\end{array}$ \\
\hline Tibial & $64^{\mathrm{a}}$ & $56^{\mathrm{a}}$ & 22 \\
Femoral & 11 & 4 & 0 \\
Double-level & 24 & 38 & $78^{\mathrm{b}}$ \\
Uncorrectable & 2 & 2 & 0 \\
\hline
\end{tabular}

Values are shown as percentages per group

mFTA mechanical femorotibial angle

${ }^{a}$ Significant difference between $3^{\circ}-5^{\circ}$ and $6^{\circ}-8^{\circ}$ mFTA $(p<0.001)$ and between $3^{\circ}-5^{\circ}$ and $\geq 9^{\circ} \mathrm{mFTA}(p=0.005)$ (Qui-square test followed by post hoc tests with Bonferroni correction)

${ }^{\mathrm{b}}$ Significant difference compared to $3^{\circ}-5^{\circ}$ and $6^{\circ}-8^{\circ} \quad(p<0.001$ and $p=0.001$ ) (Qui-square test followed by post hoc tests with Bonferroni correction)

by two clinical studies: Akamatsu et al. [1] have shown that patients with a postoperative mMPTA $>95^{\circ}$ had worse knee function at 2 years after medial open-wedge HTO. Furthermore, Schuster et al. [47] found inferior long-term functional outcome in patients with a postoperative mMPTA $>95^{\circ}$ at 10 years after medial open-wedge HTO. On the other hand, Goshima et al. [17] reported that an overcorrected mMPTA of $>95^{\circ}$ did not affect the clinical outcome after a minimum follow-up of 2 years. It is important to note, that a mMPTA of $95^{\circ}$ does not necessarily imply joint line obliquity of $5^{\circ}$. More specifically, it has been demonstrated that changes of joint line obliquity are smaller than changes of the mMPTA because of compensatory changes in the hip and ankle joints $[17,29,38]$. Whereas a certain amount of overcorrection seems to be acceptable, further studies are necessary to better understand the interaction between MMPTA and joint 
line obliquity and their impact on outcomes after realignment osteotomies.

To avoid excessive overcorrection of the MMPTA and joint line obliquity, detailed deformity analysis and precise planning of the osteotomy are paramount [5, 43]. Several methods have been proposed, with most of them being based on mFTA measurement or the Mikulicz line [12, 31]. However, based on the findings of the present study, deformity analysis should include measurement of the knee base angles (mMPTA and mLDFA) to determine the origin of the varus deformity. This study found that only $28 \%$ of patients with varus malalignment had a tibial-based deformity. Most patients $(45 \%)$ did not show a bony deformity based on the malalignment test of Paley. In those patients, varus malalignment is either the result of intraarticular wear and/or lateral ligament laxity, or the result of small deviations of the knee base angles in both the femur and tibia. Especially patients with varus due to intraarticular wear are no good candidates for corrective osteotomies and should best be treated with unicompartmental knee arthroplasty. On the other hand, some patients may be good candidates for corrective osteotomies, despite the lack of a true bony deformity. However, a high proportion of these patients will require a double-level osteotomy to avoid an oblique joint line. The same is true in patients with severe varus malalignment despite a bony deformity $[36,45]$. In the present study, a double-level osteotomy was considered ideal in $63 \%$ if anatomic correction was intended and in 33\% if overcorrection of the mMPTA to $95^{\circ}$ was accepted. Furthermore, a double-level osteotomy was significantly more often necessary in knees with sever varus malalignment. The concept of double-level osteotomy has been introduced to restore physiologic alignment and knee base angles [5, 36, 41, 45]. Whereas older studies have observed poor results and unacceptable high complication rates [46], more recent studies have shown that double-level osteotomy is a safe procedure which enables accurate and consistent deformity correction with good clinical results and low failure rates [5, 36, 41, 45]. Nevertheless, doublelevel osteotomies are technically demanding and more invasive compared to isolated HTO. Furthermore, comparative studies between HTO and double-level osteotomies are lacking. Further studies are, therefore, necessary to prove the advantage of double-level osteotomies.

According to the results of the present study, $8 \%$ of varus deformities should be corrected at the femur via isolated DFO. However, only limited data exists about the clinical efficiency of DFO in varus knees. Van der Woude et al. [51] analyzed the results of closed-wedge valgus DFO in 15 patients with a mean age of 45 years. After a mean follow-up of 40 months, the authors reported clinical improvement and accurate correction [51]. However, from a biomechanical point of view, it must be noted, that DFO decreases tibiofemoral contact pressure more effectively in extension compared with increasing angles of knee flexion [53]. Based on knowledge of total knee arthroplasty, HTO should decrease tibiofemoral contact pressure throughout flexion angles [53]. Therefore, it remains unknown whether DFO is as effective as HTO. To date, only one study has reported results after both, DFO and HTO to correct varus malalignment. Fürmetz et al. [16] prospectively evaluated 25 consecutive patients undergoing realignment osteotomy of a varus deformity. HTO was performed in 17 patients and DFO in 11 patients. After a mean follow-up of 47 months, improvement in all clinical scores was observed, without differences between the two techniques [16]. However, the small patient cohort and relatively short follow-up period limit the conclusion of this study. Since most of the weightbearing occurs during the stance phase of gait in full extension [53], the authors believe that valgus DFO may be as effective as HTO. However, further comparative studies are necessary to proof this assumption.

This study has several limitations which must be considered. First, patients were included based on radiographs without taken clinical symptoms into consideration. Therefore, our analysis must be regarded as a cross section of varus alignment in general. It remains unknown whether the geometry of a varus knee differs with regard to the specific pathologies. However, to obtain a representative cohort for corrective osteotomies, patients $<18$ and $>60$ years were excluded. Second, alignment was measured on radiographs, which only represents a $2 \mathrm{D}$ projection of a threedimensional structure. Utilization of 3D-reconstructed CT images may, therefore, be more accurate, since bony landmarks can be determined more precisely [22]. Nevertheless, standing full-leg radiographs are the current standard for the assessment of coronal limb in the clinical practice [13]. Third, varus malalignment was defined as a mechanical tibiofemoral varus angle of $\geq 3^{\circ}$ whereas other authors may consider $\geq 5^{\circ}$ as an indication for realignment osteotomies. However, indications have evolved during the last decade and realignment osteotomies are nowadays considered in patients with $<5^{\circ}$ [35], especially when performed as a concomitant procedure with cartilage repair procedures $[6,7,33]$. The authors, therefore, believe that patients with only mild varus deformity are also important to consider. Fourth, all osteotomies were simulated to a postoperative alignment of $2^{\circ}$ of mechanical valgus. However, there is no consensus about the "ideal" postoperative alignment and an individualized approach based on the indication for osteotomy has been proposed [15]. Nevertheless, a target of $2^{\circ}-3^{\circ}$ of mechanical valgus is common in the literature [50]. Fifth, the method used for osteotomy simulation does only take static alignment into consideration. Changes of JLCA and compensatory changes of the hip and ankle joints are not included. Nevertheless, these parameters may influence postoperative knee joint obliquity, as discussed above. 
Furthermore, in knees with a large preoperative JLCA, overcorrection may have occurred with our simulation technique and less bony correction would have been required to obtain the target postoperative alignment [28]. However, it remains unclear to what extent preoperative JLCA changes after valgus osteotomy. Some studies have shown that the absolute changes after HTO are small, with differences in mean JLCA $\leq 1^{\circ}[2,28]$. Given these small changes in JLCA and the fact that $82 \%$ of our patients had a JLCA of only $0^{\circ}-3^{\circ}$, we do not belief that differences in JLCA introduced a major bias. Nevertheless, further developments in computer-based osteotomy planning should take dynamic variables into consideration.

Despite these limitations, this study underlines the importance of meticulous deformity analysis and precise osteotomy planning. Varus malalignment should not uniformly be considered a tibial-based deformity and hence be corrected via HTO. Instead, an individualized approach is recommended. A computer-based planning software which is able to simulate postoperative values for MMPTA and mLDFA is of great advantage to define the ideal osteotomy level [43]. In addition, computer-based planning software allows simulation of double-level osteotomies, which is difficult with conventional planning methods. If no dedicated planning software is available, conventional methods can be utilized such as the Miniaci method [31]. However, before osteotomy planning, mFTA and knee base angles must be measured and it must be estimated if the intended correction can be achieved via isolated HTO. Furthermore, we recommend to measure the resulting mMPTA to control for excessive overcorrection.

\section{Conclusion}

Less than one-third of patients with varus malalignment $\geq 3^{\circ}$ have a tibial deformity with $\mathrm{mMPTA}<85^{\circ}$. If anatomic correction (mMPTA $\leq 90^{\circ}, \mathrm{mLDFA} \geq 85^{\circ}$ ) is intended, only $12 \%$ of patients can be corrected via isolated HTO, whereas $63 \%$ of patients would require a double-level osteotomy. If slight overcorrection at the tibial side is accepted (mMPTA $\leq 95^{\circ}$ ), $57 \%$ of patients can be corrected via isolated HTO, whereas $33 \%$ of patients would require a double-level osteotomy, and $8 \%$ should be corrected via isolated DFO.

Acknowledgements Open Access funding provided by Projekt DEAL.

Author contributions MJF and PML designed the study. PML and MJF collected data. MJF and PWW performed the statistical analysis and wrote the manuscript. GB, JM, and PF helped to design the study, assisted with statistical analysis and data interpretation, and critically reviewed the manuscript. ABI conceived of the study, helped with data interpretation and critically reviewed the manuscript. All authors read and approved the final manuscript.

\section{Compliance with ethical standards}

Conflict of interest All authors declare that they have no conflict of interest related to this study.

Funding No funding was received.

Ethical approval Ethical approval was obtained from the Ethics Committee of the technical University Munich. All procedures performed were in accordance with the ethical standards of the institutional and/or national research committee and with the 1964 Declaration of Helsinki and its later amendments or comparable ethical standards.

Open Access This article is licensed under a Creative Commons Attribution 4.0 International License, which permits use, sharing, adaptation, distribution and reproduction in any medium or format, as long as you give appropriate credit to the original author(s) and the source, provide a link to the Creative Commons licence, and indicate if changes were made. The images or other third party material in this article are included in the article's Creative Commons licence, unless indicated otherwise in a credit line to the material. If material is not included in the article's Creative Commons licence and your intended use is not permitted by statutory regulation or exceeds the permitted use, you will need to obtain permission directly from the copyright holder. To view a copy of this licence, visit http://creativecommons.org/licenses/by/4.0/.

\section{References}

1. Akamatsu Y, Kumagai K, Kobayashi H, Tsuji M, Saito T (2018) Effect of increased coronal inclination of the tibial plateau after opening-wedge high tibial osteotomy. Arthroscopy 34(7):21582169 e 2152

2. Akasaki Y, Mizu-Uchi H, Hamai S, Tsushima H, Kawahara S, Horikawa T, Nakashima Y (2019) Patient-specific prediction of joint line convergence angle after high tibial osteotomy using a whole-leg radiograph standing on lateral-wedge insole. Knee Surg Sports Traumatol Arthrosc. https://doi.org/10.1007/s00167-01905821-8 [published online ahead of print, 2019 Dec 11]

3. Amis AA (2013) Biomechanics of high tibial osteotomy. Knee Surg Sports Traumatol Arthrosc 21(1):197-205

4. Babis GC, An KN, Chao EY, Larson DR, Rand JA, Sim FH (2008) Upper tibia osteotomy: long term results-realignment analysis using OASIS computer software. J Orthop Sci 13(4):328-334

5. Babis GC, An KN, Chao EY, Rand JA, Sim FH (2002) Double level osteotomy of the knee: a method to retain joint-line obliquity. Clinical results. J Bone Joint Surg Am 84(8):1380-1388

6. Bode G, Ogon P, Pestka J, Zwingmann J, Feucht M, Sudkamp N, Niemeyer P (2015) Clinical outcome and return to work following single-stage combined autologous chondrocyte implantation and high tibial osteotomy. Int Orthop 39(4):689-696

7. Bode G, Schmal H, Pestka JM, Ogon P, Sudkamp NP, Niemeyer P (2013) A non-randomized controlled clinical trial on autologous chondrocyte implantation (ACI) in cartilage defects of the medial femoral condyle with or without high tibial osteotomy in patients with varus deformity of less than 5 degrees. Arch Orthop Trauma Surg 133(1):43-49

8. Bonasia DE, Dettoni F, Sito G, Blonna D, Marmotti A, Bruzzone M, Castoldi F, Rossi R (2014) Medial opening wedge high tibial 
osteotomy for medial compartment overload/arthritis in the varus knee: prognostic factors. Am J Sports Med 42(3):690-698

9. Brouwer RW, Huizinga MR, Duivenvoorden T, van Raaij TM, Verhagen AP, Bierma-Zeinstra SM, Verhaar JA (2014) Osteotomy for treating knee osteoarthritis. Cochrane Database Syst Rev 12:CD004019

10. Coventry MB (1965) Osteotomy of the upper portion of the tibia for degenerative arthritis of the knee. A preliminary report. J Bone Joint Surg Am 47:984-990

11. Coventry MB (1987) Proximal tibial varus osteotomy for osteoarthritis of the lateral compartment of the knee. J Bone Joint Surg Am 69(1):32-38

12. Dugdale TW, Noyes FR, Styer D (1992) Preoperative planning for high tibial osteotomy. The effect of lateral tibiofemoral separation and tibiofemoral length. Clin Orthop Relat Res 274:248-264

13. Eberbach H, Mehl J, Feucht MJ, Bode G, Sudkamp NP, Niemeyer $P$ (2017) Geometry of the valgus knee: contradicting the dogma of a femoral-based deformity. Am J Sports Med 45(4):909-914

14. El-Azab HM, Morgenstern M, Ahrens P, Schuster T, Imhoff AB, Lorenz SG (2011) Limb alignment after open-wedge high tibial osteotomy and its effect on the clinical outcome. Orthopedics 34(10):e622-e628

15. Feucht MJ, Minzlaff P, Saier T, Cotic M, Sudkamp NP, Niemeyer P, Imhoff AB, Hinterwimmer S (2014) Degree of axis correction in valgus high tibial osteotomy: proposal of an individualised approach. Int Orthop 38(11):2273-2280

16. Furmetz J, Patzler S, Wolf F, Degen N, Prall WC, Soo C, Bocker $\mathrm{W}$, Thaller PH (2020) Tibial and femoral osteotomies in varus deformities-radiological and clinical outcome. BMC Musculoskelet Disord 21(1):201

17. Goshima K, Sawaguchi T, Shigemoto K, Iwai S, Fujita K, Yamamuro Y (2019) Comparison of clinical and radiologic outcomes between normal and overcorrected medial proximal tibial angle groups after open-wedge high tibial osteotomy. Arthroscopy 35(10):2898-2908 e2891

18. Hankemeier S, Gosling T, Richter M, Hufner T, Hochhausen C, Krettek C (2006) Computer-assisted analysis of lower limb geometry: higher intraobserver reliability compared to conventional method. Comput Aid Surg 11(2):81-86

19. Harris JD, McNeilan R, Siston RA, Flanigan DC (2013) Survival and clinical outcome of isolated high tibial osteotomy and combined biological knee reconstruction. Knee 20(3):154-161

20. Hernigou P, Duffiet P, Julian D, Guissou I, Poignard A, Flouzat-Lachaniette CH (2013) Outcome of total knee arthroplasty after high tibial osteotomy: does malalignment jeopardize the results when using a posterior-stabilized arthroplasty? HSS J 9(2):134-137

21. Hess S, Moser LB, Amsler F, Behrend H, Hirschmann MT (2019) Highly variable coronal tibial and femoral alignment in osteoarthritic knees: a systematic review. Knee Surg Sports Traumatol Arthrosc 27(5):1368-1377

22. Hirschmann MT, Hess S, Behrend H, Amsler F, Leclercq V, Moser LB (2019) Phenotyping of hip-knee-ankle angle in young non-osteoarthritic knees provides better understanding of native alignment variability. Knee Surg Sports Traumatol Arthrosc 27(5):1378-1384

23. Hirschmann MT, Moser LB, Amsler F, Behrend H, Leclercq V, Hess S (2019) Phenotyping the knee in young non-osteoarthritic knees shows a wide distribution of femoral and tibial coronal alignment. Knee Surg Sports Traumatol Arthrosc 27(5):1385-1393

24. Hui C, Salmon LJ, Kok A, Williams HA, Hockers N, van der Tempel WM, Chana R, Pinczewski LA (2011) Long-term survival of high tibial osteotomy for medial compartment osteoarthritis of the knee. Am J Sports Med 39(1):64-70
25. Issin A, Sahin V, Kockara N, Gursu SS, Kurtuldu A, Yildirim $\mathrm{T}$ (2012) Is proximal tibia the major problem in varus gonarthrosis? Evaluation of femur and ankle. Eklem Hastalik Cerrahisi 23(3):128-133

26. Jackson JP, Waugh W, Green JP (1969) High tibial osteotomy for osteoarthritis of the knee. J Bone Joint Surg Br 51(1):88-94

27. Kellgren JH, Lawrence JS (1957) Radiological assessment of osteo-arthrosis. Ann Rheum Dis 16(4):494-502

28. Lee DH, Park SC, Park HJ, Han SB (2016) Effect of soft tissue laxity of the knee joint on limb alignment correction in open-wedge high tibial osteotomy. Knee Surg Sports Traumatol Arthrosc 24(12):3704-3712

29. Lee KM, Chang CB, Park MS, Kang SB, Kim TK, Chung CY (2015) Changes of knee joint and ankle joint orientations after high tibial osteotomy. Osteoarthritis Cartilage 23(2):232-238

30. Lobenhoffer P, Agneskirchner JD (2003) Improvements in surgical technique of valgus high tibial osteotomy. Knee Surg Sports Traumatol Arthrosc 11(3):132-138

31. Miniaci A, Ballmer FT, Ballmer PM, Jakob RP (1989) Proximal tibial osteotomy. A new fixation device. Clin Orthop Relat Res 246:250-259

32. Minzlaff P, Feucht MJ, Saier T, Cotic M, Plath JE, Imhoff AB, Hinterwimmer S (2016) Can young and active patients participate in sports after osteochondral autologous transfer combined with valgus high tibial osteotomy? Knee Surg Sports Traumatol Arthrosc 24(5):1594-1600

33. Minzlaff P, Feucht MJ, Saier T, Schuster T, Braun S, Imhoff AB, Hinterwimmer S (2013) Osteochondral autologous transfer combined with valgus high tibial osteotomy: long-term results and survivorship analysis. Am J Sports Med 41(10):2325-2332

34. Moser LB, Hess S, Amsler F, Behrend H, Hirschmann MT (2019) Native non-osteoarthritic knees have a highly variable coronal alignment: a systematic review. Knee Surg Sports Traumatol Arthrosc 27(5):1359-1367

35. Na YG, Lee BK, Hwang DH, Choi ES, Sim JA (2018) Can osteoarthritic patients with mild varus deformity be indicated for high tibial osteotomy? Knee 25(5):856-865

36. Nakayama H, Iseki T, Kanto R, Kambara S, Kanto M, Yoshiya S, Schroter S (2020) Physiologic knee joint alignment and orientation can be restored by the minimally invasive double level osteotomy for osteoarthritic knees with severe varus deformity. Knee Surg Sports Traumatol Arthrosc 28(3):742-750

37. Nakayama H, Schroter S, Yamamoto C, Iseki T, Kanto R, Kurosaka K, Kambara S, Yoshiya S, Higa M (2018) Large correction in opening wedge high tibial osteotomy with resultant joint-line obliquity induces excessive shear stress on the articular cartilage. Knee Surg Sports Traumatol Arthrosc 26(6):1873-1878

38. Oh KJ, Ko YB, Bae JH, Yoon ST, Kim JG (2016) Analysis of knee joint line obliquity after high tibial osteotomy. J Knee Surg 29(8):649-657

39. Paley D, Herzenberg JE, Tetsworth K, McKie J, Bhave A (1994) Deformity planning for frontal and sagittal plane corrective osteotomies. Orthop Clin North Am 25(3):425-465

40. Paley D, Pfeil J (2000) Principles of deformity correction around the knee. Orthopade 29(1):18-38

41. Saragaglia D, Blaysat M, Mercier N, Grimaldi M (2012) Results of forty two computer-assisted double level osteotomies for severe genu varum deformity. Int Orthop 36(5):999-1003

42. Saragaglia D, Mercier N, Colle PE (2010) Computer-assisted osteotomies for genu varum deformity: which osteotomy for which varus? Int Orthop 34(2):185-190

43. Schroter S, Elson DW, Ateschrang A, Ihle C, Stockle U, Dickschas J, Harrer J (2017) Lower limb deformity analysis and the planning of an osteotomy. J Knee Surg 30(5):393-408 
44. Schroter S, Ihle C, Mueller J, Lobenhoffer P, Stockle U, van Heerwaarden R (2013) Digital planning of high tibial osteotomy. Interrater reliability by using two different software. Knee Surg Sports Traumatol Arthrosc 21(1):189-196

45. Schroter S, Nakayama H, Yoshiya S, Stockle U, Ateschrang A, Gruhn J (2019) Development of the double level osteotomy in severe varus osteoarthritis showed good outcome by preventing oblique joint line. Arch Orthop Trauma Surg 139(4):519-527

46. Schuller HM, van Dijk CN, Fidler MW (1987) Poor results of double osteotomy for the rheumatoid knee. Acta Orthop Scand 58(3):253-255

47. Schuster P, Gesslein M, Schlumberger M, Mayer P, Mayr R, Oremek D, Frank S, Schulz-Jahrsdorfer M, Richter J (2018) Ten-year results of medial open-wedge high tibial osteotomy and chondral resurfacing in severe medial osteoarthritis and varus malalignment. Am J Sports Med 46(6):1362-1370

48. Thienpont E, Schwab PE, Cornu O, Bellemans J, Victor J (2017) Bone morphotypes of the varus and valgus knee. Arch Orthop Trauma Surg 137(3):393-400

49. Tischer T, Paul J, Pape D, Hirschmann MT, Imhoff AB, Hinterwimmer S, Feucht MJ (2017) The impact of osseous malalignment and realignment procedures in knee ligament surgery: a systematic review of the clinical evidence. Orthop J Sports Med 5(3):2325967117697287
50. Van den Bempt M, Van Genechten W, Claes T, Claes S (2016) How accurately does high tibial osteotomy correct the mechanical axis of an arthritic varus knee? A systematic review. Knee 23(6):925-935

51. van der Woude JA, Spruijt S, van Ginneken BT, van Heerwaarden RJ (2016) Distal femoral valgus osteotomy: bone healing time in single plane and biplanar technique. Strategies Trauma Limb Reconstr 11(3):177-186

52. Verdonk PC, Demurie A, Almqvist KF, Veys EM, Verbruggen G, Verdonk R (2005) Transplantation of viable meniscal allograft. Survivorship analysis and clinical outcome of one hundred cases. J Bone Joint Surg Am 87(4):715-724

53. Wylie JD, Scheiderer B, Obopilwe E, Baldino JB, Pavano C, Macken CJ, Bell R, Mazzocca AD, Arciero RA, Imhoff FB (2018) The effect of lateral opening wedge distal femoral varus osteotomy on tibiofemoral contact mechanics through knee flexion. Am J Sports Med 46(13):3237-3244

Publisher's Note Springer Nature remains neutral with regard to jurisdictional claims in published maps and institutional affiliations.

\section{Affiliations}

\section{Matthias J. Feucht ${ }^{1,2}$ D $\cdot$ Philipp W. Winkler ${ }^{1} \cdot$ Julian Mehl $^{1} \cdot$ Gerrit Bode $^{2} \cdot$ Philipp Forkel $^{1} \cdot$ Andreas B. Imhoff $^{1}$. Patricia M. Lutz ${ }^{1}$}

1 Department for Orthopedic Sports Medicine, Technical University Munich, Ismaninger Str. 22, 81675 Munich, Germany
2 Department of Orthopedics and Trauma Surgery, Medical Center, Faculty of Medicine, Albert-Ludwigs-University of Freiburg, Freiburg, Germany 